\title{
Lack of sample diversity in research on adolescent depression and social media use: a scoping review and meta-analysis
}

\author{
Sakshi Ghai ${ }^{*}{ }^{1,2}$ \\ University of Cambridge \\ Faisal Awadh ${ }^{4}$ \\ University of Cambridge
}

\author{
Luisa Fassi ${ }^{* 2,3}$ \\ University of Cambridge \\ Amy Orben ${ }^{2}$ \\ University of Cambridge
}

Research on whether social media use relates to adolescent depression is rapidly increasing. However, is it adequately representing the diversity of global adolescent populations? We conducted a pre-registered scoping review (research published between 2018-2020; 34 articles) to investigate the proportion of studies recruiting samples from the Global North vs. Global South and assess whether the association between social media and depression varies depending on the population being studied. Sample diversity was lacking between regions: 70\% of studies examined Global North populations. The link between social media and depression was positive and significant in the Global North but null and non-significant in the Global South. There was also little evidence of diversity within regions in both sampling choices and reporting of participants' demographics. Given that most adolescents live in the Global South and sample diversity is crucial for the generalisability of research findings, urgent action is needed to address these oversights.

Keywords: social media, depression, sample diversity, adolescents, Global North, Global South

Half of the world's population is digitally connected (World Economic Forum, 2017), and mobile phone ownership is common even for people living in the most deprived regions (World Development Report, 2016). This development has allowed many adolescents to begin accessing social media, fundamentally changing the way

\footnotetext{
*Joint first authorship. ${ }^{1}$ Department of Psychology, University of Cambridge; ${ }^{2}$ MRC Cognition and Brain Sciences Unit, University of Cambridge; ${ }^{3}$ Department of Psychiatry, University of Cambridge; ${ }^{4}$ School of Clinical Medicine, University of Cambridge.

Sakshi Ghai $\odot$ https://orcid.org/0000-0002-8488-0273

Luisa Fassi $\odot$ https://orcid.org/0000-0002-0520-6425

Faisal Awadh $\odot$ https://orcid.org/0000-0003-0023-3157

Amy Orben 10 https://orcid.org/0000-0002-2937-4183

We have no conflict of interest to disclose. Correspondence concerning this article should be addressed to: Sakshi Ghai, University of Cambridge, Department of Psychology, Downing Street, Cambridge, CB2 3EB. Email: sg912@cam.ac.uk
}

they live, communicate and interact with educational or clinical services (Livingstone et al., 2017). In parallel, there has been a rise in concern across researchers, clinicians and policymakers that social media use might be driving an increase in adolescent depression or a decline in their wellbeing (Twenge et al., 2018; Twenge \& Campbell, 2019; Spears et al., 2015; Salmela-Aro et al., 2017).

However, such links remain complex and heavily debated, as studies have mainly been correlational, finding mixed results (Odgers \& Jensen, 2020) with small effect sizes (Orben \& Przybylski, 2019). Thus far, the lack of consensus has mostly been ascribed to individual differences such as gender, socioeconomic status, and personality traits influencing the link between social media use and depression or wellbeing (Odgers \& Jensen, 2020). Surprisingly, despite the renewed focus on how these effects may differ from 'adolescents to 
adolescent' (Beyens et al., 2020), little to no attention has been given to how the association of social media and depression might vary in diverse, traditionally underrepresented and understudied communities, for example, populations living in the Global South. Such a lack of sample diversity in psychological science has been broadly recognised (Henrich et al., 2010; Hruschka et al., 2018; Rad et al., 2018). For instance, only $7 \%$ of research samples published in Psychological Science in 2017 represented nearly $85 \%$ of the world's population (Rad et al., 2018). Furthermore, clinical trials often solely recruit samples from the Global North and high-resource settings (Polo et al., 2019; Hall, 2001).

The dearth of sample diversity in psychological and clinical research poses a significant challenge to the generalisability of research findings. As Rosmarin (2016) highlights: "Unless we start to embrace the fact that not all human beings are alike, clinical science will become increasingly irrelevant to most of the population". Notably, the cultural contexts in which adolescents live heavily shape their beliefs, and the prevalence of stigma influences perceptions of depressive symptomatology in the Global South (Altweck et al., 2015). Furthermore, how social media is accessed, what platforms are used and how adolescents interact with them varies substantially across contexts and populations around the world (Miller, 2019). Therefore, to make claims about social media use and depression that are relevant to all global populations, we need to understand whether 1) there is sufficient sample diversity to generalise existing results, and 2) there are any differences in how social media relates to depression between regions.

The issue of sample diversity is twofold. On the one hand, as highlighted above, it concerns the lack of diverse representation across geographic areas (e.g., the Global South). On the other hand, it also relates to the lack of recruiting diverse samples within regions. Research often relies on convenient, unrepresentative, and homogenous groups (e.g., white, male, educated, middle-class participants) (Henrich et al., 2010; Gurven, 2019), not capturing the diversity present within a certain country or region. Moreover, participants' demographics such as ethnicity, income and education are routinely not measured even in clinical trials, and studies often show high variability in how participants' demographics are described, making it challenging to gauge sample diversity when reviewing the existing literature (Crosby et al., 2010). For example, a recent review of 342 randomised control trials of depression over 36 years revealed that, despite improving over time, most studies did not report ethnicity and socioeconomic indicators. Additionally, clinical trials were far more likely to exclude, rather than include, linguistic minorities, and did not test Asian American, Native Hawaiian/Pacific Islander, Native American/Native Alaskan and multi-ethnic samples (Polo et al., 2019).

Such lack of sample diversity will directly impact the validity and reliability of research findings and likely lead to more inaccurate generalisations (Hall, 2001). This is especially relevant in policyand clinically relevant research areas, for example, the research on the potential links between social media use and adolescent depression, in which results derived from a sub-group of the population might inform policy or clinical decisions that impact other groups traditionally under-represented in psychological research.

\section{The Current Study}

Given that more than two-thirds of the world's adolescents live in the Global South (Azzopardi et al., 2019), we need to investigate the extent to which research quantifying the links between adolescent social media use and depression can be generalised to diverse populations. Furthermore, we need to understand whether there are similar issues on a within-region level, where populations routinely under-studied (e.g., disadvantaged groups) in academic research may also be overlooked. To tackle these issues, we hereby examine whether research samples are representative of the world's adolescent population, whether the association between depression and social media use differs depending on the global population being studied and whether research samples sufficiently 
represent within-region diversity. Overall, we aim to address generalisability and representativeness issues in this heavily expanding research area.

We conducted a pre-registered scoping review examining if studies quantifying the link between adolescent social media use and depression, published in the last three years, relied on skewed or diverse samples at two levels: between and within regions. First, to understand the between-region diversity, we analysed the extent to which studies relied only on samples from the Global North and then tested whether the meta-correlation between depression and social media use differed for studies sampling populations from the Global North vs. the Global South. Second, to gauge the extent of within-region diversity, we considered whether studies tested samples that included disadvantaged groups and were representative of the general population, as well as investigated whether studies reported participants' demographics beyond age and gender (e.g., education, income, and ethnicity).

\section{Methods}

As research findings on the impact of social media use across diverse populations are limited, we adopted a scoping review methodology (Arksey and O'Malley, 2005). In comparison to systematic reviews, scoping reviews do not aim to provide a critically appraised and synthesised answer to a particular question. Instead, they aim to identify knowledge gaps in the literature and offer an overview of existing evidence. Scoping reviews have been increasingly used in medicine (Barclay \& Hilton, 2019; Agarwal et al., 2019), clinical psychiatry (Jacobsen et al., 2018; Leijten et al., 2021; Reinders et al., 2019) and most recently in psychology (Premachandra \& Lewis, 2021). Similarly to systematic reviews, scoping reviews use rigorous and transparent ways to analyse the evidence base on a topic (Munn et al., 2018). For instance, they include exhaustive searches for resources and extract data in a reproducible and structured way. Our pre-registered scoping review was executed using a five-stage methodological framework outlined by Arksey and O'Malley (2005): 1) Identifying the research question; 2) Identifying relevant studies; 3) Study selection; 4) Charting the data; 5) Collating, summarising, and reporting the results. In addition, we followed the guidelines proposed by the Preferred Reporting Items for Systematic Reviews and Meta-Analyses (PRISMA) extension for scoping reviews (PRISMA-ScR) checklist (Tricco et al., 2018). Lastly, we performed all analyses with the $\mathrm{R}$ statistical programming language (version 4.0.4). The packages used in the analysis are listed in our OSF project.

\section{Identifying the Research Question}

The present review addresses whether 1) the current research into social media's relation with depression has sufficiently diverse samples between global regions (Global North vs. Global South); 2) the link between social media use and depression differs based on global region; 3) the samples collected are representative and/or under-studied (e.g., disadvantaged groups); and 4) there is adequate reporting of participants' demographics to gauge other forms of diversity.

\section{Identifying Relevant Studies}

\section{Search Terms}

Our search terms, which we applied using a title and abstract search, included items targeting social media, depression, and adolescent populations combined using the "AND” operator (details on the search string provided in Supplementary Table C, D, E). We included both general social media search terms (OR: "social media", "online social networking", "internet use", "social-media", "online-community", "online-communities", "social-app", "social-apps", "social-networking-app", "social-networking-apps", "social-networkingsite", "social-networking-sites") and the 15 most popular social media websites worldwide (Statista, 2021) (OR: "Facebook", "YouTube", "WhatsApp", "Messenger", "WeChat", "Instagram", "QQ", "Tumblr", "Qzone", "Tik-Tok", "Sina-Weibo", "Twitter", "Reddit", "BaiduTieba", "LinkedIn"). We also included search terms related to depression (OR: "depression", "depressive disorder", "depression", "depressed", "depressive") and adolescence (OR: "adolescent", "child", "adolescent development", "adolescent", 
"adolescence", "teen", "teens", "teenager", "teenagers", "youth", "youths", "young", "young-adult", "young-adults", "student", "students", "undergraduate", "postgraduate").

\section{Inclusion Criteria}

To identify relevant studies, we applied a variety of search criteria. Firstly, we searched for articles published in the last three years (20182020), a time window that allowed us to capture the exponential growth in social media research worldwide. Secondly, we searched for studies of adolescents, defined as those between the ages of 10 and 24 (Sawyer et al., 2018). Third, we used three databases for our literature search: PubMed, PsycInfo, and Global Health. These spanned different academic fields intending to prioritise both medical and psychological research. Articles were selected by screening the title and abstract; however, when a decision could not be made based on this, the article was read at full length.

The initial search found 460 non-duplicate items (Figure 1), which we then reviewed, applying different inclusion criteria. Firstly, only peer-reviewed research was included. Secondly, we only considered articles using empirical or mixed-methodological approaches (with quantitative analyses focusing on the relationship between social media use and depression) published from 2018 to 2020. Dissertations, non-peer review articles, and articles that did not include empirical work (e.g., systematic reviews, literature reviews, metaanalyses, meta-synthesis, scoping reviews, narrative reviews, rapid reviews, or qualitative approaches) were excluded. Thirdly, we only included articles that utilised at least one quantitative measure of each variable: depression, social media use and the relationship between social media use and depression. Finally, we did not exclude studies based on language; most retrieved studies were written in English except for one study written in Persian.

\section{Study Selection}

FA retrieved 611 articles (across all three databases) and exported them to Rayyan (Ouzzani et al., 2016). At this stage, duplicates were identified and removed, leaving 460 non-duplicate citations to be screened. Following, FA and AO screened the studies' titles/abstracts according to the inclusion/exclusion criteria. As a result, 408 articles were excluded, 52 articles were retrieved, of which 3 were marked as 'maybe include', and 21 conflicts were identified (often those studies with abstracts that poorly described the study measurements). To resolve this, articles were full text screened and discussed in depth until both FA and AO came to a unanimous agreement. As a result, 18 articles were further excluded, bringing the final inclusion count to 34 articles (Figure 1).

\section{Figure 1.}

Flowchart of the study selection process

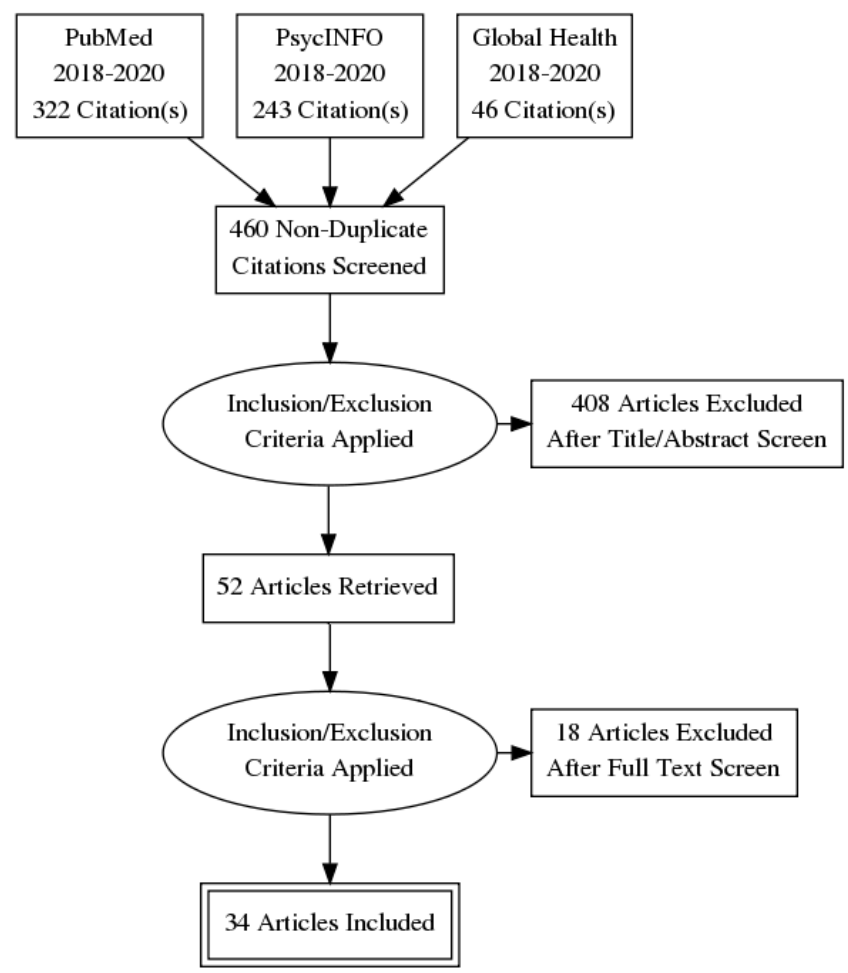

Note. The chart depicts the number of identified, screened and included articles in the present scoping review. 


\section{Charting the Data}

Using a pre-specified coding spreadsheet, LF coded all articles, while SG coded a random selection of $25 \%$ of articles. Out of the articles coded twice, over 95\% agreement was reached. The coding spreadsheet included the title, year, journal, authors, study design (i.e., cross-sectional or longitudinal), and geographical areas (specifically whether the samples were collected in the Global North vs. Global South and the country). To code the geographical areas, we employed the regional classifications provided by the United Nation's specialised agency, the official source for Information and Technology statistics (International Telecommunications Union, 2021). If the geographical location of the studied sample was not mentioned, we used the primary author affiliation as a proxy. Furthermore, the coding spreadsheet included the depression and social media use measures used in each study (open response: type of measure and name of the employed questionnaire); and the correlation reported between these measurements (for more details about variations in measurement, see Supplementary Table A and B).

We also coded whether the study collected a convenient, representative and/or disadvantaged sample (dichotomous; yes/no) and whether the study reported participants' demographics such as age, gender, education, ethnicity, and income (dichotomous; reported/not reported). A study was coded as using a representative sample if authors directly mentioned that the study was representative, or they used probability sampling. On the contrary, we coded a study as using a convenient sample if the authors tested student populations or otherwise collected participant data via non-probability sampling. Further, disadvantaged samples were operationalised in two ways: a) if the sample study mentioned economic disadvantage (i.e., income or unemployment), educational disadvantage, family disadvantage (i.e., family not finished school), health disadvantage (i.e., mental, or physical illness), and environmental disadvantage (i.e., dirty surroundings or neighbourhoods), or b) if the study mentioned a disadvantaged sample that the authors defined themselves. Finally, we also coded whether the study mentioned any caveat associated with the sample they recruited (i.e., small sample size, lack of ethnic diversity, limitations to generalizability across populations) and noted the citation counts of each paper (see Supplementary Materials for more details). We also provide details in the Supplementary Materials on why we deviated from our original plan of coding these studies along the WEIRD acronym to determine whether the samples of the study were white, educated, industrialised, rich, and democratic (Henrich et al., 2010).

\section{Meta-analysis}

We conducted an exploratory meta-analysis to test whether the correlation between social media use and depression differed in studies that tested samples from the Global North vs. the Global South. The association between the two variables was defined as positive when increased social media use was associated with an increase in depressive symptomatology. For all analyses, we employed an a priori statistical significance level of $\alpha=0.05$.

Among the selected studies, some reported an effect size other than a correlation coefficient (e.g., a regression coefficient) to describe the association of social media use and depression. Hence, we first included studies that reported a correlation coefficient in the article main text or supplementary materials $(N=22)$. If a study reported an effect size other than a correlation, the authors were contacted by email and asked to provide a correlation coefficient (e.g., Pearson's $r$ or Spearman's rs). LF and SG contacted 12 authors, of which 6 replied, providing the coefficient(s) of interest. The remaining 6 studies were excluded from the metaanalysis. The data file available on OSF provides detailed information regarding which authors were contacted.

Raw effect sizes were transformed into normalised correlation coefficients (Fisher's $z$ ) to stabilise the variances. For effect sizes initially reported as Spearman's $r s$, we first transformed them to $r$ and then transformed them to Fisher's $z$. We used the following equation to perform this transformation, $r=2 \sin (\operatorname{rs}(\pi / 6))$, as reported by Rupinski and Dunlap (2016) and employed in recently published 
literature (Parry et al., 2021). All correlations included in the meta-analysis were transformed from Fisher's $z$ back to Pearson's $r$ for reporting.

\section{Meta-correlation Model}

A random-effects model was applied to calculate overall summary effect sizes. To interpret the outcomes of the correlational meta-analyses, in line with Cohen (1988), we took correlation coefficients of 0.1 to be small, 0.30 to be medium, and 0.50 or greater to be large effect sizes. Of note, some studies contributed to a larger extent to the meta-analysis because they reported multiple effect sizes. Thus, to account for variance inflation emerging from dependent observations for different measures for the same participants, we used cluster-robust variance estimation (RVE) based on the sandwich method with adjusted estimators for small samples and correlated effects weighting scheme. A default value of $r=0.80$ was specified in the RVE (Hedges et al., 2010; Fisher \& Tipton, 2015). To examine the variance and heterogeneity among the considered effect sizes, we computed $Q$ and $I^{2}$, interpreting statistically significant $Q$ values to indicate heterogeneity and $I^{2}$ values of approximately $25 \%, 50 \%$ and $75 \%$ to indicate low, moderate, and high heterogeneity, respectively.

\section{Moderator Analysis}

We applied a moderator analysis to examine the contribution of the global area (Global North/ Global South) as a source of heterogeneity in the observed correlations. This variable satisfied the minimum requirement of four effect sizes per moderator level, which was defined based on published literature (Parry et al., 2021). Given our in- terest in disentangling how the association of social media use and depression may differ between the Global North and the Global South, for this moderator, in addition to meta-regression models, we estimated separate random-effects models to produce summary effect sizes for each subgroup.

\section{Results}

In this study, we investigated whether a lack of sample diversity skews research on the association between adolescent depression and social media use. To that end, we examined 1) if the Global South is adequately represented in the existing literature, 2) how the link between depression and social media differs depending on whether the Global North or Global South is studied, 3) whether there is sufficient sample diversity within-regions, as indicated by the use of disadvantaged and non-convenient samples and 4) whether the reporting of participants' demographics was enough to assess sample diversity.

\section{Sample Diversity Between Regions}

Out of the 34 considered studies, 70\% (24 studies) examined participants from the Global North, while only $30 \%$ (10 studies) examined a population from the Global South (Figure 2). Within the Global North, a wide range of countries were represented: Canada, the US, the UK, Belgium, Netherlands, Norway, Iceland, Japan, and Australia. Most studies were from the US (26\%; 9 studies), followed closely by Europe (26\%; 9 studies) and Oceania (15\%; 5 studies). Within the Global South, we found only 3\% (1 study) from Latin America (Mexico), 9\% (3 studies) from the Middle East (Jordan/Lebanon/Iran), 18\% (6 studies) from Asia (China/Bangladesh/India) excluding Japan and no studies representing Africa. 


\section{Figure 2.}

Cartogram showing the size of each country's adolescent population in relation to the percentage of studies found in our scoping review



Note. This cartogram represents a resized world map based on the adolescent population aged 10-24 years living in each global region, based on data retrieved from the United Nation (Department of Economic and Social Affairs, Population Division, 2020). The darker blue areas represent more densely populated regions, while the lighter blue areas represent less populated regions. Further, we display a percentage break-down of the geographical region of each study included in our scoping review. Specifically, the majority of studies tested Western samples - 26\% studies constitute North America, while 26\% from Europe and 15\% from Oceania. In addition, 21\% of the studies comprise an Asian sample (including Japan), 9\% from the Middle East, a fraction of the studies from Latin America (3\%) and not a single study from Africa. Although Japan falls under Asia, it was coded as Global North because it is a high-income country, as specified by the International Telecommunications Union (2021). This cartogram demonstrates the geographical bias of research towards Global North samples.

Hence, the over-reliance on Global North samples suggests that the majority of adolescent populations around the world are not well-represented within this research area.

To examine if the link between depression and social media differed depending on whether the population being studied was from the Global North or South, we ran a meta-correlation including 40 effect sizes from 28 studies. Across these comparisons, the total sample size was 83066. On average, a comparison involved 2077 participants $(S D=$ 3612, $M D=909, M I N=102, M A X=16398)$. We calculated meta-correlation coefficients separately for effect sizes from the Global South (7 effect sizes from 7 studies) and the Global North (33 effect sizes from 21 studies). The effect size for the Global North was significant yet small ( $r=0.16$, 
95\% CI 0.13-0.20, $P<$. 001). On the contrary, the and non-significant $(r=0.08,95 \%$ CI -0.14-0.29, effect size for the Global South was close to zero $P=.426)$.

\section{Figure 3.}

Forest plot of effect sizes on the association between social media use and depression

\begin{tabular}{|c|c|c|}
\hline & \multirow{2}{*}{$\begin{array}{c}\text { Correlation }[95 \% \mathrm{Cl}] \\
-0.19[-0.30,-0.08]\end{array}$} \\
\hline & $-1-1$ & \\
\hline Al-Dwaikat et al., 2020 & $\mathrm{H}=\mathrm{H}$ & $-0.11[-0.20,-0.02]$ \\
\hline Zeeni et al., 2018 & $\mapsto-1$ & $-0.11[-0.23,0.02]$ \\
\hline Fardouly et al., 2020.2 & $\mapsto-1$ & $-0.03[-0.12,0.06]$ \\
\hline Fardouly et al., 2020.3 & $H=-1$ & $-0.00[-0.09,0.08]$ \\
\hline Heffer et al. , 2019.2 & \pm & $0.02[-0.03,0.07]$ \\
\hline Gillig et al., 2020 & $\mapsto-1$ & $0.03[-0.10,0.16]$ \\
\hline Fardouly et al., 2018 & $\mapsto-1$ & $0.04[-0.08,0.16]$ \\
\hline Ferguson, 2021 & * & $0.04[0.02,0.06]$ \\
\hline Vannucci et al., 2019.4 &  & $0.05[-0.01,0.11]$ \\
\hline Jasso-Medrano et al., 2018 & $H=H$ & $0.07[-0.03,0.17]$ \\
\hline Vannucci et al., 2019.3 & 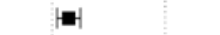 & $0.08[0.02,0.14]$ \\
\hline Vannucci et al., 2019.5 & 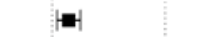 & $0.08[0.02,0.14]$ \\
\hline Vannucci et al., 2019.2 & $1 \pm$ & $0.09[0.03,0.15]$ \\
\hline \multicolumn{2}{|l|}{ Muzaffar et al., 2018} & $0.10[-0.09,0.29]$ \\
\hline \multicolumn{2}{|l|}{ Barthorpe et al., 2020} & $0.11[0.08,0.14]$ \\
\hline \multicolumn{2}{|l|}{ Heffer et al. , 2019.1} & $0.13[0.08,0.18]$ \\
\hline \multicolumn{2}{|l|}{ Frison et al., 2020.1} & $0.13[0.08,0.18]$ \\
\hline \multicolumn{2}{|l|}{ Vannucci et al., 2019.1} & $0.13[0.07,0.19]$ \\
\hline \multicolumn{2}{|l|}{ Ellis et al., 2020.1} & $0.14[0.08,0.20]$ \\
\hline \multicolumn{2}{|l|}{ Vannucci et al., 2019.6} & $0.14[0.08,0.19]$ \\
\hline \multicolumn{2}{|l|}{ Fardouly et al., 2020.1} & $0.15[0.07,0.23]$ \\
\hline \multicolumn{2}{|l|}{ Padilla-Walker et al., 2020} & $0.15[0.09,0.21]$ \\
\hline \multicolumn{2}{|l|}{ Rodgers et al., 2020} & $0.15[0.08,0.22]$ \\
\hline \multicolumn{2}{|l|}{ Frison et al., 2020.2} & $0.16[0.11,0.21]$ \\
\hline \multicolumn{2}{|l|}{ Hawes et al., 2020} & $0.16[0.09,0.23]$ \\
\hline \multicolumn{2}{|l|}{ Choukas-Bradley et al., 2019} & $0.17[0.06,0.27]$ \\
\hline \multicolumn{2}{|l|}{ Dredge et al., 2020} & $0.17[0.06,0.27]$ \\
\hline \multicolumn{2}{|l|}{ Heffer et al., 2019.2} & $0.18[0.10,0.25]$ \\
\hline \multicolumn{2}{|l|}{ Thorisdottir et al., 2019} & $0.20[0.18,0.22]$ \\
\hline \multicolumn{2}{|l|}{ Niu et al., 2018} & $0.21[0.14,0.27]$ \\
\hline \multicolumn{2}{|l|}{ Houghton et al., 2018} & $0.22[0.17,0.26]$ \\
\hline \multicolumn{2}{|l|}{ Kelly et al., 2018} & $0.22[0.20,0.24]$ \\
\hline \multicolumn{2}{|l|}{ Thorisdottir et al., 2020} & $0.23[0.19,0.27]$ \\
\hline \multicolumn{2}{|l|}{ Ellis et al., 2020.2} & $0.24[0.18,0.30]$ \\
\hline \multicolumn{2}{|l|}{ Twenge et al., 2020} & $0.25[0.23,0.27]$ \\
\hline \multicolumn{2}{|l|}{ Heffer et al., 2019.1} & $0.26[0.18,0.33]$ \\
\hline \multicolumn{2}{|l|}{ Brunborg et al., 2019} & $0.32[0.25,0.38]$ \\
\hline Aalbers et al., 2019 & $\longmapsto \hookrightarrow$ & $0.38[0.22,0.53]$ \\
\hline Deimazar et al., 2019 & $\mathrm{H}=\mathrm{H}$ & $0.46[0.39,0.53]$ \\
\hline RE Model with RVE (using correlational weighting scheme & $\boldsymbol{\theta}$ & $0.14[0.09,0.20]$ \\
\hline 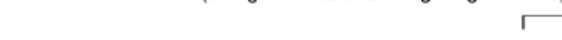 & $\begin{array}{l}1 \\
1\end{array}$ & \\
\hline-0.4 & $0 \quad 0.2$ & \\
\hline
\end{tabular}

Note. Individual Pearson's $r$ coefficients are depicted as filled squares with the size indicating the relative weight of each effect size estimate in the meta-analysis. The colour indicates the global area, namely: red squares for samples from the Global South and black squares for samples from the Global North. The filled black diamond represents the overall summary effect size across all studies $(r=0.14,95 \% C I 0.09-0.20, P<0.001)$, calculated using robust variance estimation to account for dependencies between effect sizes coming from the same study. The error bars and diamond width represent the 95\%CIs for the effect sizes. The dashed reference line at the intercept for $r=0.5$ represents the point from which the magnitude of the association would be sufficient to conclude that the association between social media use and depression is moderate. 
We found a high degree of heterogeneity in the considered correlation coefficients $(Q$ (39) $=$ 738.87, $P<0.001$ ), indicating that the examined studies did not share a common effect size, ranging both from positive to negative, and from strong to weak associations. This was further confirmed by the RVE analysis: $T 2=0.014, I 2=96.31 \%$ (for a more detailed explanation on heterogeneity and publication bias refer to Supplementary Materials: Supplementary Figure 1 and Meta-analysis Specifications). To identify potential outliers, we conducted both outlier and influence diagnostics (extrapolating Cook's distance, covariance ratios and diagonal elements of the hat matrix) with the 'metafor' package (Viechtbauer, 2010). Influence diagnostics indicated one outlier $(I D=11$, are $a=$ Global South, $r=0.462$, 95\%CI $0.39-0.53$, residuals =3.30). A sensitivity analysis excluding this outlier produced a summary effect size for the Global South similar to the original analysis $(r=0.01, C I$ -0.17-0.18, $P=.910$ ). Therefore, this study was included in the follow-up moderator analysis.

Given our interest in examining the role of global areas as a potential source of heterogeneity and given the availability of sufficient data (Global South, $k=7$; Global North, $k=33$ ), we ran a moderator analysis. We found a negative non-significant result on the moderating role of Global South vs. Global North areas on the association between adolescent depression and social media use $(\beta=-0.08$, $S E=0.09,95 \% C I-0.29-0.13, P=0.41)$. Therefore, we cannot currently conclude that global area is a significant source of variability in the association between social media use and depression. Notably, despite following standard procedures, the statistical power of the moderator analyses was limited by the number of available studies. Given that only a small and heterogeneous number of effect sizes (7/40) was reported for the Global South, confidence intervals capturing the meta-coefficient for this level of the moderator were very large.

\section{Sample Diversity Within Regions}

To examine the extent to which research findings are generalisable within countries, we investigated 1) the sampling characteristics of the recruited participants and 2) whether studies adequately reported their demographics (Figure 4).

To ensure diverse representation across research samples, we specifically focused on the use of convenient, representative, and disadvantaged samples (Panel A, Figure 4). Our results showed that most studies used convenience samples across the two regions: $80 \%$ of samples from the Global South were convenient, compared to $62 \%$ from the Global North. A limited number of studies tested representative populations (e.g., using probability sampling): specifically, 33\% of samples from the Global North compared to $10 \%$ from the Global South. Lastly, $4 \%$ of samples from the Global North were disadvantaged compared to $0 \%$ from the Global South.

We also examined whether studies provided sufficient detail to judge sample diversity within-regions (Panel B, Figure 4). We found that information about gender and age was reported by all studies, irrespective of geographical area. Education was reported by most studies, specifically, $90 \%$ of studies from the Global South reported information about adolescents' current educational level, compared to $75 \%$ of studies from the Global North. On the contrary, information on income and 


\section{Figure 4.}

Bar graph displaying the proportion of papers in relation to the recruited samples and the reported participants' demographics
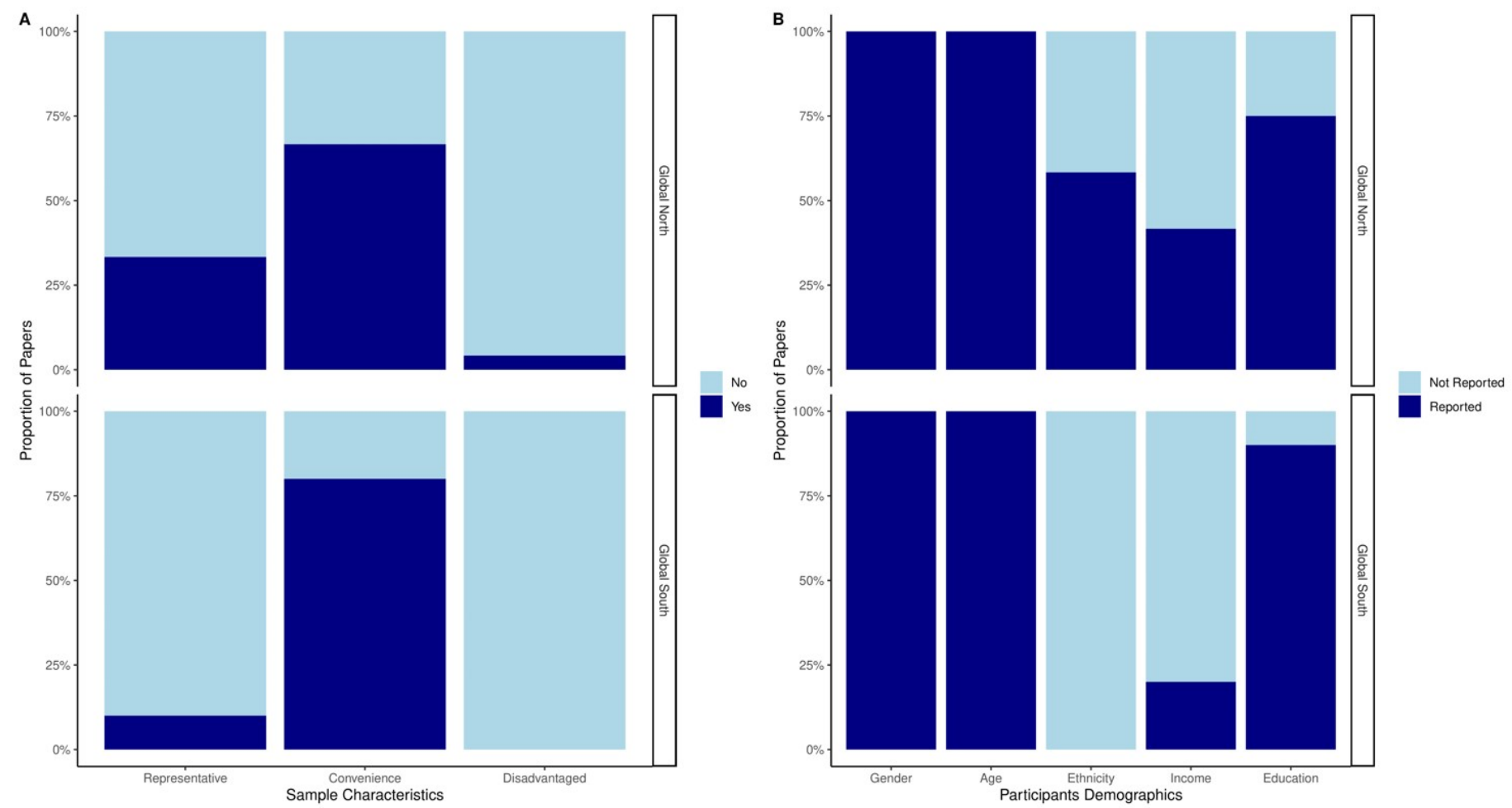

Note. The bar graphs show differences in the extent to which A) Studies from the Global North compared to the Global South recruited samples that were representative, convenient, and disadvantaged, on the left panel; and B) Studies from the Global North compared to the Global South reported participants' demographics (gender, age, ethnicity, income, and education), on the right panel.

ethnicity was not always reported. Specifically, $42 \%$ of studies from the Global North reported information about income. This percentage was even lower for studies from the Global South (20\%). Furthermore, $58 \%$ of studies from the Global North mentioned information about participants' ethnicity compared to $0 \%$ of studies from the Global South. In conclusion, studies both within the Global North and Global South did not comprehensively report the socio-demographic diversity of adolescents.

\section{Discussion}

The evident lack of diverse samples in clinical psychological research has been widely documented
(Coakley et al., 2012). In the current study, we draw attention to the way this issue may bias an area of research that is currently undergoing exponential growth and is closely related to clinical and policy applications around the world, namely: the study of social media use and adolescent depression.

Our findings show that 1) a striking $70 \%$ of reviewed studies recruited samples from the Global North, while only $30 \%$ of studies recruited samples from the Global South; 2) the meta-correlation between depression and social media use was small, positive, and significant in samples from the Global North, while it is null and non-significant in samples from the Global South; 3) studies from 
both the Global North and Global South heavily relied on convenient and non-representative samples, with little research being carried out on disadvantaged adolescent populations; 4) while age and gender were always reported, education was often reported, ethnicity and income were missing in the majority of studies.

Consistent with previous reviews examining the extensive lack of sample diversity in psychological research (Arnett, 2008; Rad et al., 2018), we found an overall lack of sample diversity across regions. It is remarkable that the Global South is not only home to the majority of the world's adolescents but also comprises the highest numbers of social media users worldwide (Statista, 2021; UN Data, 2020). Hence, the lack of sample diversity in existing research can strongly bias our understanding of how social media use is associated with adolescents' depression. This is especially the case as attitudes towards depression and technology use vary widely between the Global North and South.

Our meta-analysis, investigating whether the association of social media use and adolescents' depression varied between the Global North and Global South, found - on average across all samples - a positive yet small correlation, a result aligned to previous literature (Orben, 2020). Among the factors that may explain such variability (e.g., gender-specific effects or methodological choices), our focus was on sample diversity, an often neglected yet crucial source of heterogeneous outcomes within clinical psychology. We found a positive and significant correlation between social media and depression in the Global North; in contrast, in the Global South, the correlation was null and non-significant. We run moderation analysis to further explore the role of global area as a potential source of heterogeneity among the examined effect sizes. In this case, our results showed that global area (Global North vs. Global South) was not a significant moderator of the meta-correlation coefficient. However, interpretation of this result requires caution, especially due to the large confidence intervals associated with the Global South meta-correlation coefficient. The broad confidence interval, spanning from a lower bound of 0.14 to an upper bound of 0.29 can be ascribed to the limited and highly heterogeneous effect sizes reflecting research from the Global South, with only 7 out of 40 effect sizes coming from this area. This, in turn, points to the existing gap in sample diversity in the literature. Hence, the paucity of research samples from the Global South does not allow us to draw adequately powered conclusions.

To further examine whether research samples provided a representative snapshot of adolescent populations, we considered the samples characteristics of each study. We found that the majority of studies relied on convenience sampling with almost negligible amounts of disadvantaged samples. These results were in a similar direction in both geographical regions, with almost no studies focusing on disadvantaged samples in the Global South. In line with previous studies on participants' demographic diversity (Hughes et al., 2016), we also found that, although gender and age were always reported, and education was often reported, ethnicity and income were rarely reported in the reviewed studies. Therefore, there was not only little variance between regions but there was also insufficient information on whether the samples were socio-economically or ethnically diverse within those regions. Furthermore, future research may also need to account for other aspects participants' demographics such as religion, sexual-orientation, or urban and rural differences.

We underscore three important caveats to our study. Firstly, despite conducting a systematic search, we restricted our time frame to three years. Hence, we could have inadvertently excluded key articles that study the relationship between social media use and depression in under-represented samples. Secondly, scoping reviews do not typically review the quality of evidence (Davis et al., 2009), and thus, we might have overlooked variability in, for example, the quality of measurements used to describe social media use as well as depression. Lastly, all the considered studies relied on correlations to capture the relationship between depression and social media use and hence, no causal inferences can be drawn regarding whether increased social media use leads to depression or vice versa. 


\section{Conclusion}

In our scoping review and meta-analysis, twothirds of reviewed studies sampled populations from the Global North. As the majority of the world's adolescent population lives in the Global South, this bias can have clinical repercussions and needs to become an urgent area of focus. However, scientists also need to expand research in the Global North to ensure that samples are not homogenous and include socially disadvantaged populations.

Sample diversity is indeed crucial to develop a nuanced perspective on adolescent depression and its relation to social media use. To account for both between and within-region variations, future research should first and foremost diversify its sampling methodologies and broaden the reporting of participants' demographics. However, to truly create a more enduring change in the field, we need to a) co-create research and collaborate with diverse researchers globally to advance the scholarship on social media use and depression and b) urgently prioritise and fund more work that advances underrepresented research samples living in both the Global North and Global South. Only by doing this will we account for sample diversity as a source of heterogeneity in research outcomes and develop generalisable research to safeguard adolescents against the possible consequences of social media use globally.

\section{Data and Analysis Code}

\section{Available on OSF at: https://osf.io/8f6tc/}

\section{Acknowledgements}

We are grateful to Tom Metherell for his advice on the cartogram and Douglas Parry for his inputs on the meta-analysis.

\section{Author Contributions}

Contributorship was documented with CRediT (Holcombe et al., 2020):

Conceptualisation: Sakshi Ghai, Faisal Awadh, and Amy Orben.

Data curation: Sakshi Ghai and Luisa Fassi. Formal analysis: Sakshi Ghai and Luisa Fassi. Methodology: Sakshi Ghai, Faisal Awadh, and Amy Orben.

Project administration: Sakshi Ghai and Amy Orben.

Supervision: Amy Orben.

Visualisation: Sakshi Ghai and Luisa Fassi. Writing - original draft: Sakshi Ghai, Luisa Fassi, Faisal Awadh, and Amy Orben.

Writing - review \& editing: Sakshi Ghai, Luisa Fassi, and Amy Orben.

\section{Funding}

SG was supported by the G.C Grindley Fund, Christ's College Bursary, and Takshila Education Society. LF was supported by the Medical Research Council as part of the Doctoral Training Program (DTP). AO was supported by an Emmanuel College Research Fellowship and the Medical Research Council. 


\section{References}

Agarwal, D., Hanafi, N. S., Chippagiri, S., Brakema, E. A., Pinnock, H., Khoo, E. M., Sheikh, A., Liew, S.-M., Ng, C.-W., Isaac, R., Chinna, K., Ping, W. L., Hussein, N. B., \& Juvekar, S. (2019). Systematic scoping review protocol of methodologies of chronic respiratory disease surveys in low/middle-income countries. Npj Primary Care Respiratory Medicine, 29(1), 17. https://doi.org/10.1038/s41533-019-0129-7

Altweck, L., Marshall, T. C., Ferenczi, N., \& Lefringhausen, K. (2015). Mental health literacy: A cross-cultural approach to knowledge and beliefs about depression, schizophrenia and generalised anxiety disorder. Frontiers in Psychology, 0, 1272. https://doi.org/10.3389/FPSYG.2015.01272

Arksey, H., \& O'Malley, L. (2007). Scoping studies: Towards a methodological framework. International Journal of Social Research Methodology, 8:1, 19:32. https://doi.org/10.1080/136455703200011961 $\underline{6}$

Arnett, J. J. (2016). The neglected 95\%: Why American psychology needs to become less American. In A. E. Kazdin (Ed.), Methodological issues and strategies in clinical research (4th ed.). (pp. 115-132). American Psychological Association. https://doi.org/10.1037/14805-008

Azzopardi, P. S., Hearps, S. J. C., Francis, K. L., Kennedy, E. C., Mokdad, A. H., Kassebaum, N. J., Lim, S., Irvine, C. M. S., Vos, T., Brown, A. D., Dogra, S., Kinner, S. A., Kaoma, N. S., Naguib, M., Reavley, N. J., Requejo, J., Santelli, J. S., Sawyer, S. M., Skirbekk, V., ... Patton, G. C. (2019). Progress in adolescent health and wellbeing: Tracking 12 headline indicators for 195 countries and territories, 1990-2016. The Lancet, 393(10176), 1101-1118.

https://doi.org/10.1016/S01406736(18)32427-9

Barclay, L., \& Hilton, G. M. (2019). A scoping review of peer-led interventions following spinal cord injury. Spinal Cord 2019 57:8, 57(8), 626-635. https://doi.org/10.1038/s41393-019-0297-x

Beyens, I., Pouwels, J. L., Driel, I. I. van, Keijsers, L., \& Valkenburg, P. M. (2020). The effect of social media on well-being differs from adolescent to adolescent. Scientific Reports 2020 10:1, 10(1), 1-11.

https://doi.org/10.1038/s41598-020-67727-7

Coakley, M., Fadiran, E. O., Parrish, L. J., Griffith, R. A., Weiss, E., \& Carter, C. (2012). Dialogues on Diversifying Clinical Trials: Successful Strategies for Engaging Women and Minorities in Clinical Trials. Journal of Women's Health, 21(7), 713-716.

https://doi.org/10.1089/jwh.2012.3733

Cohen, J. Statistical Power Analysis for the Behavioral Sciences (Academic, 1988).

Crosby, R. A., Salazar, L. F., DiClemente, R. J., \& Lang, D. L. (2010). Balancing rigor against the inherent limitations of investigating hardto-reach populations. Health Education Research, 25(1), 1-5. https://doi.org/10.1093/her/cyp062

Davis, K., Drey, N., \& Gould, D. (2009). What are scoping studies? A review of the nursing literature. International Journal of Nursing Studies, 46(10), 1386-1400.

https://doi.org/10.1016/j.ijnurstu.2009.02.010

Fisher, Z., \& Tipton, E. (2015). robumeta: An Rpackage for robust variance estimation in meta-analysis. https://arxiv.org/abs/1503.02220v1

Hall, G. C. N. (2001). Psychotherapy research with ethnic minorities: Empirical, ethical, and conceptual issues. Journal of Consulting and Clinical Psychology, 69(3), 502-510. https://doi.org/10.1037/0022-006X.69.3.502

Hedges, L. V., Tipton, E., \& Johnson, M. C. (2010). Robust variance estimation in metaregression with dependent effect size estimates. Research Synthesis Methods, 1(1), 3965. https://doi.org/10.1002/JRSM.5

Henrich, J., Heine, S. J., \& Norenzayan, A. (2010). The weirdest people in the world? Behavioral and Brain Sciences, 33(2-3), 61-83. https://doi.org/10.1017/S0140525X0999152X

Holcombe, A. O., Kovacs, M., Aust, F., \& Aczel, B. (2020). Documenting contributions to scholarly articles using CRediT and tenzing. PLOS ONE, 15(12), e0244611. https://doi.org/10.1371/journal.pone.0244611

Hruschka, D. J., Medin, D. L., Rogoff, B., \& Henrich, J. (2018). Pressing questions in the study of psychological and behavioral diversity. Proceedings of the National Academy of 
Sciences, 115(45), 11366-11368.

https://doi.org/10.1073/PNAS.1814733115

Hughes, J. L., Camden, A. A., \& Yangchen, T.

(2016). Rethinking and updating demographic questions: Guidance to improve descriptions of research samples [Editorial]. Psi Chi Journal of Psychological Research, 21(3), 138151.

International Telecommunications Union. Retrieved November 23, 2021, from

https://meta.wikimedia.org/wiki/List_of_countries_by_regional_classification

Jacobsen, P., Hodkinson, K., Peters, E., \& Chadwick, P. (2018). A systematic scoping review of psychological therapies for psychosis within acute psychiatric in-patient settings. The British Journal of Psychiatry, 213(2), 490-497. https://doi.org/10.1192/bjp.2018.106

Livingstone, S., Nandi, A., Banaji, S. and Stoilova, M. (2017) Young adolescents and digital media: uses, risks and opportunities in low-and middle-income countries: a rapid evidence review. London: Gender and Adolescence: Global Evidence.

Leijten, P., Weisz, J. R., \& Gardner, F. (2021). Research Strategies to Discern Active Psychological Therapy Components: A Scoping Review. Clinical Psychological Science, 9(3), 307-

322. https://doi.org/10.1177/21677026209786 $\underline{15}$

Miller, D. (2019). Anthropological Studies of Mobile Phones. Technology and Culture, 60(4), 1093-1097.

https://doi.org/10.1353/tech.2019.0103

Munn, Z., Peters, M. D. J., Stern, C., Tufanaru, C., McArthur, A., \& Aromataris, E. (2018). Systematic review or scoping review? Guidance for authors when choosing between a systematic or scoping review approach. $B M C$ Medical Research Methodology, 18(1), 143. https://doi.org/10.1186/s12874-018-0611-x

Odgers, C. L., \& Jensen, M. R. (2020). Annual Research Review: Adolescent mental health in the digital age: Facts, fears, and future directions. Journal of Child Psychology and Psychiatry, 61(3), 336-348. https://doi.org/10.1111/JCPP.13190

Orben, A. (2020). Teenagers, screens and social media: A narrative review of reviews and key studies. Social Psychiatry and Psychiatric Epidemiology 2020 55:4, 55(4), 407-414.

https://doi.org/10.1007/S00127-019-01825-4

Orben, A., \& Przybylski, A. K. (2019). The association between adolescent wellbeing and digital technology use. Nature Human Behaviour 2019 3:2, 3(2), 173-182.

https://doi.org/10.1038/s41562-018-0506-1

Ouzzani, M., Hammady, H., Fedorowicz, Z., \&

Elmagarmid, A. (2016). Rayyan-A web and mobile app for systematic reviews. Systematic Reviews 2016 5:1, 5(1), 1-10.

https://doi.org/10.1186/S13643-016-0384-4

Parry, D. A., Davidson, B. I., Sewall, C. J. R., Fisher, J. T., Mieczkowski, H., \& Quintana, D. S. (2021). A systematic review and metaanalysis of discrepancies between logged and self-reported digital media use. Nature $\mathrm{Hu}$ man Behaviour, 5(11), 1535-1547. https://doi.org/10.1038/s41562-021-01117-5

Polo, A. J., Makol, B. A., Castro, A. S., ColónQuintana, N., Wagstaff, A. E., \& Guo, S. (2019). Diversity in randomised clinical trials of depression: A 36-year review. Clinical Psychology Review, 67, 22-35. https://doi.org/10.1016/J.CPR.2018.09.004

Premachandra, B., \& Lewis, N. A. (2021). Do We Report the Information That Is Necessary to Give Psychology Away? A Scoping Review of the Psychological Intervention Literature 20002018. Perspectives on Psychological Science . https://doi.org/10.1177/1745691620974774

Rad, M. S., Martingano, A. J., \& Ginges, J. (2018). Toward a psychology of Homo sapiens: Making psychological science more representative of the human population. Proceedings of the National Academy of Sciences, 115(45), 11401-11405.

https://doi.org/10.1073/PNAS.1721165115

Reinders, N. J., Branco, A., Wright, K., Fletcher, P. C., \& Bryden, P. J. (2019). Scoping Review: Physical Activity and Social Functioning in Young People With Autism Spectrum Disorder. Frontiers in Psychology, 10, 120. https://doi.org/10.3389/fpsyg.2019.00120

Rosmarin, D. H. (2016). Diversity Science: New Dawn in a Golden Age. Clinical Psychological Science, 4(4), 701-703. https://doi.org/10.1177/2167702616647926

Rupinski, M. T., \& Dunlap, W. P. (2016). Approximating Pearson Product-Moment Correlations from Kendall's Tau and Spearman's 
Rho, 56(3), 419-429.

https://doi.org/10.1177/001316449605600300 4

Salmela-Aro, K., Upadyaya, K., Hakkarainen, K., Lonka, K., \& Alho, K. (2016). The Dark Side of Internet Use: Two Longitudinal Studies of Excessive Internet Use, Depressive Symptoms, School Burnout and Engagement Among Finnish Early and Late Adolescents. Journal of Youth and Adolescence 2016 46:2, 46(2), 343-357.

https://doi.org/10.1007/S10964-016-0494-2

Sawyer, S. M., Azzopardi, P. S., Wickremarathne, D., \& Patton, G. C. (2018). The age of adolescence. The Lancet Child \& Adolescent

Health, 2(3), 223-228.

https://doi.org/10.1016/S2352-

4642(18)30022-1

Spears, B. A., Taddeo, C. M., Daly, A. L., Stretton, A., \& Karklins, L. T. (2015). Cyberbullying, help-seeking and mental health in young Australians: Implications for public health. International Journal of Public Health 2015 60:2, 60(2), 219-226.

https://doi.org/10.1007/S00038-014-0642-Y

Statista | Facebook users by country 2021. Retrieved November 20, 2021, from https://www.statista.com/statistics/268136/top-15-countries-based-on-number-of-facebook- users/

Tricco, A. C., Lillie, E., Zarin, W., O'Brien, K. K., Colquhoun, H., Levac, D., Moher, D., Peters, M. D. J., Horsley, T., Weeks, L., Hempel, S., Akl, E. A., Chang, C., McGowan, J., Stewart, L., Hartling, L., Aldcroft, A., Wilson, M. G., Garritty, C., ... Straus, S. E.
(2018). PRISMA Extension for Scoping Reviews (PRISMA-ScR): Checklist and Explanation., 169(7), 467-473.

https://doi.org/10.7326/M18-0850

Twenge, J. M., \& Campbell, W. K. (2019). Media Use Is Linked to Lower Psychological WellBeing: Evidence from Three Datasets. Psychiatric Quarterly 2019 90:2, 90(2), 311-331. https://doi.org/10.1007/S11126-019-09630-7

Twenge, J. M., Joiner, T. E., Rogers, M. L., \& Martin, G. N. (2017). Increases in Depressive Symptoms, Suicide-Related Outcomes, and Suicide Rates Among U.S. Adolescents After 2010 and Links to Increased New Media Screen Time: Clinical Psychological Science, 6, 6(1), 3-17. https://doi.org/10.1177/2167702617723376

Twenge, J. M., Martin, G. N., \& Campbell, W. K. (2018). Decreases in psychological wellbeing among American adolescents after 2012 and links to screen time during the rise of smartphone technology. Emotion, 18(6), 765780. https://doi.org/10.1037/EMO0000403

Viechtbauer, W. (2010). Conducting meta-analyses in R with the metafor package. Journal of Statistical Software, 36(3), 1-48. https://doi.org/10.18637/JSS.V036.I03

World Development Report 2016: Digital Dividends. (2016). World Development Report 2016: Digital Dividends. https://doi.org/10.1596/978-1-4648-0671-1

Adolescent demographics. UNICEF DATA. (2019, October 27). Retrieved 22 November, 2021, from https://data.unicef.org/topic/adolescents/demographics/. 